RELIGIOUS PARENTING 



\section{Religious Parenting}

TRANSMITTING FAITH

AND VALUES IN

CONTEMPORARY AMERICA

Christian Smith, Bridget Ritz

छ Michael Rotolo 
Copyright (C) 2020 by Princeton University Press

Requests for permission to reproduce material from this work

should be sent to permissions@press.princeton.edu

Published by Princeton University Press

41 William Street, Princeton, New Jersey 08540

6 Oxford Street, Woodstock, Oxfordshire OX20 1TR

press.princeton.edu

All Rights Reserved

Library of Congress Cataloging-in-Publication Data

Names: Smith, Christian, 196o- author. | Ritz, Bridget, 1992- author. |

Rotolo, Michael, 1991- author.

Title: Religious parenting : transmitting faith and values in contemporary

America / Christian Smith, Bridget Ritz, Michael Rotolo.

Description: Princeton, New Jersey : Princeton University Press, 2020. |

Includes bibliographical references and index.

Identifiers: LCCN 2019027047 (print) | LCCN 2019027048 (ebook) |

ISBN 9780691194967 (hardback : acid-free paper) | ISBN 9780691197821 (ebook)

Subjects: LCSH: Parenting-Religious aspects. | Religion and sociology-United States. |

Parental influences-United States. | Intergenerational communication-Religious

aspects. | Intergenerational relations-Religious aspects.

Classification: LCC BL625.8 .S53 2020 (print) | LCC BL625.8 (ebook) | DDC 204/.41-dc23

$\mathrm{LC}$ record available at https://lccn.loc.gov/2019027047

LC ebook record available at https://lccn.loc.gov/2019027048

British Library Cataloging-in-Publication Data is available

Editorial: Fred Appel and Jenny Tan

Production Editorial: Karen Carter

Jacket/Cover Design: Amanda Weiss

Production: Erin Suydam

Publicity: Tayler Lord and Kathryn Stevens

This book has been composed in Miller

Printed on acid-free paper. $\infty$

Printed in the United States of America

$\begin{array}{llllllllll}10 & 9 & 8 & 7 & 6 & 5 & 4 & 3 & 2 & 1\end{array}$ 
For Brian

-B.J.R.

For Olivia

-M.S.R.

For Helen (R.I.P.)

- C.S.S. 
